\title{
Performance of fingerprint quality measures depending on sensor technology
}

\author{
Fernando Alonso-Fernandez* \\ Biometric Recognition Group - ATVS, \\ EPS, Universidad Autonoma de Madrid, \\ Campus de Cantoblanco - C/ Francisco Tomas y Valiente 11, 28049 Madrid, Spain.
}

Fabio Roli, Gian Luca Marcialis ${ }^{\dagger}$

Dept. of Electrical and Electronic Eng. - University of Cagliari, Piazza d'Armi, 09123 Cagliari, Italy

Julian Fierrez, Javier Ortega-Garcia, Joaquin Gonzalez-Rodriguez ${ }^{\ddagger}$

(Dated: November 15, 2007)

\begin{abstract}
Although many image quality measures have been proposed for fingerprints, few works have taken into account how differences among capture devices impact on the image quality. In this paper, several representative measures for assessing the quality of fingerprint images are compared using an optical and a capacitive sensor. We implement and test a representative set of measures that rely on different fingerprint image features for quality assessment. The capability to discriminate between images of different quality and its relationship with the verification performance is studied. For our verification experiments, we use a minutiae- and a ridge-based matcher, which are the most common approaches for fingerprint recognition. We report differences depending on the sensor, and interesting relationships between sensor technology and features used for quality assessment are also pointed out.
\end{abstract}

PACS numbers: 42.30.Sy, 87.57.C-, 87.57.N-, 95.75.Mn

\footnotetext{
*Electronic address: fernando.alonso@uam.es; This work has been carried out while F. A.-F. was guest scientist at the University of Cagliari

${ }^{\dagger}$ Electronic address: \{roli, marcialis\}@diee.unica.it

${ }^{\ddagger}$ Electronic address: \{julian.fierrez, javier.ortega, joaquin.gonzalez\}@uam.es; Also at Biometric
} 
Recognition Group - ATVS 


\section{INTRODUCTION}

Fingerprints are commonly used to verify the identity of a person with high accuracy $[1,2]$. Recent experimental results pointed out that the verification performance is affected by the quality of the image provided by the electronic sensor $[3,4]$. Quality in biometric systems is a current research challenge [5] and even the best fingerprint verification systems worldwide struggle in the presence of noisy images, as demonstrated in the FVC2004 benchmark [6]. A significant drop of performance was observed in FVC2004 with respect to the previous edition in 2002 [7] due to deliberate quality corruption of the databases introduced during the acquisition. In the last FVC2006 edition [8], no deliberate difficulties were introduced in the acquisition, but the population is more heterogeneous, including manual workers and elderly people. Also, no constraints were enforced to guarantee a minimum quality in the acquired images and the final datasets were selected from a larger database by choosing the most difficult fingers according to a quality index, to make the benchmark sufficiently difficult for an evaluation.

So far, several capture devices have been proposed for acquiring fingerprint images [1]. Among them, optical and capacitive sensors are the most widely used. They are based on different physical principles (see Fig. 1). In optical sensors, the finger touches a glass prism and the prism is illuminated with diffused light. The light is reflected at the valleys and absorbed at the ridges. The reflected light is focused onto a CCD or CMOS sensor. On the other hand, capacitive sensors use the electrical properties of the skin as the second armature of the capacitor formed against the silicon acquisition surface. Due to their different physical principles, the conditions affecting the quality of the acquired images are expected to be different for optical and capacitive sensors. It has been demonstrated in several studies that changing the sensor has impact in the verification performance, not only in fingerprint systems [9-12], but also in other biometric traits [13, 14]. The effect of factors such as pressure on the sensor, dryness/wetness of the skin, dirt, noise, residual prints on the sensor, etc. are expected to impact differently during the fingerprint acquisition. Therefore, it can be hypothesized that the two sensors provide images of different quality in many cases. In addition, one can think that the degradation of the verification performance with the quality can be different for the two sensors. Many quality measures for fingerprint images acquired from electronic sensors have been proposed in the literature [15]. Existing measures rely 
on different features of fingerprint images for quality assessment. However, few works have taken into account how differences among fingerprint capture devices impact on the quality measure computation $[10,16,17]$. In our opinion, some measures could be suitable for the optical sensor and not for the capacitive one, and vice-versa.

\section{iiijFigure 1 goes here i¿id}

Accordingly, the main goal of this paper is to compare by experiments some representative state-of-the-art measures for assessing the quality of fingerprint images, in order to provide indications on which of them are best suited for a certain capture device. An optical and a capacitive sensor are used in our experiments. We evaluate a set of quality measures in terms of their capability to discriminate among images of different quality and their relationship with the verification performance. We use in our verification experiments a minutiae- and a ridge-based matcher, which are the most widely used approaches for fingerprint verification [1]. Reported results show differences depending on the sensor or on the matcher, and relationships between sensor technology and features used for quality assessment are also pointed out. The scope of our conclusions is obviously limited by the fact that we adopt a particular commercial sensor for each family (optical and capacitive). However, it should be noted that the acquisition principle is the same for all optical and capacitive sensors, so it is possible that reported results could be confirmed in a next and larger experimental stage with other commercial sensors based on optical and capacitive acquisition principles.

The rest of the paper is organized as follows. Section II describes the quality measures used in our study. In Section III, we describe our experiments and results, and conclusions are finally drawn in Section IV.

\section{iiiiFigure 2 goes here $i ¿ i ¿$}

\section{QUALITY MEASURES FOR OPTICAL AND CAPACITIVE FINGERPRINT IMAGES}

A number of approaches for fingerprint image quality computation have been described in the literature. A taxonomy is given in [15] (see Fig. 2). Image quality is assessed by measuring one of the following properties: ridge strength or directionality, ridge continuity, ridge clarity, integrity of the ridge-valley structure, or estimated verification performance when using the image at hand. A number of sources of information are used to measure 
these properties: i) angle information provided by the direction field, ii) Gabor filters, which represent another implementation of the direction angle [18], iii) pixel intensity of the gray-scale image, $i v$ ) power spectrum, and $v$ ) Neural Networks. Fingerprint quality can be assessed either analyzing the image in a holistic manner, or combining the quality from local non-overlapped blocks of the image.

In the following, we give some details about the quality measures used in this paper. Different measures have been selected from the literature in order to have a representative set. We have implemented several measures that make use of the above mentioned properties for quality assessment, see Table I:

\section{A. Ridge-strength measures}

- Orientation Certainty Level $\left(Q_{O C L}\right)$ [19], which measures the energy concentration along the dominant direction of ridges using the intensity gradient. A relative weight is given to each region of the image based on its distance from the centroid, since regions near the centroid are supposed to provide more reliable information [20].

- Standard deviation of Gabor filter responses $\left(Q_{G A B O R}\right)$ [21]. The fingerprint image is filtered using a Gabor filter with $m$ different directions. For fingerprint regions with good quality (i.e. strong ridge orientation), one or several filter responses are larger than the others. The standard deviation of the $m$ filter responses is then used to determine the quality.

- Energy concentration in the power spectrum $\left(Q_{E N E R G Y}\right)$ [20], which is computed using ring-shaped bands. For a fingerprint image, the ridge frequency value lies within a certain range and it is expected that as fingerprint image quality increases, the energy will be more concentrated in ring patterns within the spectrum.

\section{B. Ridge-continuity measures}

- Local Orientation Quality $\left(Q_{L O Q}\right)$ [22], which is computed as the average absolute difference of direction angle with the surrounding image blocks, providing information about how smoothly direction angle changes from block to block. 
- Continuity of the orientation field $\left(Q_{C O F}\right)[19]$. This method rely on the fact that, in good quality images, ridges and valleys must flow sharply and smoothly in a locally constant direction. The direction change along rows and columns of the image is examined. Abrupt direction changes between consecutive blocks are then accumulated and mapped into a quality score.

\section{Ridge-clarity measures}

- Local Clarity Score $\left(Q_{L C S}\right)$ [22]. The sinusoidal-shaped wave that models ridges and valleys (e.g. see [23]) is used to segment ridge and valley regions. The clarity is then defined as the overlapping area of the gray level distributions of segmented ridges and valleys. For ridges/valleys with good clarity, both distributions should have a very small overlapping area.

- Amplitude and variance of the sinusoid that models ridges and valleys $\left(Q_{A}\right.$ and $\left.Q_{V A R}\right)$ [23]. Based on these parameters, blocks are classified as good and bad. The quality of the fingerprint is then computed as the percentage of foreground blocks marked as good.

\section{Ridge-integrity measures}

- Ridge frequency and ridge-to-valley thickness ratio $\left(Q_{F R E C}\right.$ and $\left.Q_{R V}\right)$ [19]. Ridges and valleys are modeled as a sinusoidal-shaped wave along the direction normal to the local ridge orientation (e.g. see [23]). Ridge frequency and ridge-to-valley thickness ratio are computed for each image block. A valid range is defined for both measures, and blocks whose measures fall outside of the range are marked as "bad" blocks.

\section{E. Measures based on estimated verification performance}

- Matcher performance $\left(Q_{N F I S}\right)$. One popular method based on classifiers $[24,25]$ defines the quality measure as the degree of separation between the match and nonmatch distributions of a given fingerprint, which is computed using Neural Networks. 
This quality assessment algorithm is included in the publicly available NIST software $[26]$.

\section{iiiiTable 1 goes here ¿i¿i \\ iiiiTable 2 goes here $¿ i ¿ i$ \\ iiiiFigure 3 goes here $i \_i \&$}

\section{EXPERIMENTAL RESULTS}

\section{A. Database description}

Similarly to the FVC2004 benchmark [6], we created a database containing 1680 images in which image quality has been artificially corrupted by using an acquisition procedure with variable contact pressure, artificial dryness and moistness. The index, middle and ring fingers of both hands from seven volunteers were acquired using an optical and a capacitive sensor (six classes per person, therefore having a total number of $6 \times 7=42$ classes per sensor). We used the Biometrika FX2000 (312 × 372 pixels images at $569 \mathrm{dpi})$ and the Precise Biometrics MC100 $(300 \times 300$ pixels images at $500 \mathrm{dpi})$ as optical and capacitive sensor, respectively (see Fig. 4). The NFIS2 matcher used in our experiments is developed for 500 dpi images, thus images from the optical sensor are first downsampled to dpi.

\section{iiiiFigure 4 goes here ¿i.ii}

First, ten impressions of each finger were acquired in normal conditions, i.e. asking users to press against the sensor in a natural way. This results in 420 multi-sensor fingerprint images, being referred from now on as the DIEEE dataset. Next, another ten impressions of each finger were acquired under corrupted quality conditions. Across the ten acquisitions, users were asked to apply low and high pressure against the sensor surface, and simulate dryness and moisture conditions. Table II show the conditions imposed to each of the ten impressions. This results in a second subset of 420 multi-sensor fingerprint images, being referred from now on as the EXTREME dataset. With this procedure, we are not aimed at study in depth the specific effect of pressure and humidity in the image quality but to obtain images of very low quality. Other studies [27] specifically study the impact fingerprint force has on image quality by measuring pressure with sensing equipments.

At the end of the data collection, we gathered two multi-sensor datasets from the same 
volunteers, one acquired in a natural way and the second having a number of sources of difficulty: artifacts due to wet fingers, poor contrast due to skin dryness or low contact pressure, distortion due to high contact pressure, etc. Examples are shown in Fig. 3.

\section{iiiiFigure 5 goes here $i \dot{i} i$}

\section{B. Separation capabilities of quality measures}

We first compare the capability of the selected quality measures to discriminate between good and bad quality images acquired with each sensor. For this purpose, three subsets were extracted for each sensor from the EXTREME dataset by visual inspection of their image qualities, one subset with 62 images of low quality, one subset with 62 images of medium quality, and one subset with 62 images of good quality. Images for each subset have been selected in order to have clearly different quality images. The following factors have been taking into account to assess the quality of fingerprint images: incomplete fingerprint (low pressure), smudged ridges in different zones of the image (high pressure or moisture), nonuniform contrast of the ridges (low pressure or dryness), background noise in the image or latent fingerprints from previous acquisitions (moisture), weak impression of the ridge structure (low pressure or dryness), and significant breaks in the ridge structure or artifacts between ridges (high pressure or moisture). These characteristics are quantified by visual inspection and only images undoubtedly classified as bad, medium or good quality are considered. Examples are shown in Fig. 3.

As evaluation criterion, the Fisher distance (FD) has been used, which is a measure of statistical separation among classes. FD is a class separation statistic working at best when distributions of random variables under study can be described by their first-order statistics. It works clearly as a sub-optimal choice when this does not happen, and nonparametric statistics should be preferred, but it is well-known that parametric statistic are difficult to estimate. It is also worth noting that FD showed to model effectively a classseparation estimation in genera cases [28]. The FD expression for two classes $A$ and $B$ is as follows:

$$
F D=\frac{\left(\mu_{A}-\mu_{B}\right)^{2}}{\sigma_{A}^{2}+\sigma_{B}^{2}}
$$

where $\mu_{A}$ and $\sigma_{A}\left(\mu_{B}\right.$ and $\left.\sigma_{B}\right)$ are the mean and variance of class $A$ (class $B$ ). 
The quality of all images from the three subsets is computed and then, the mean quality value and standard deviation value of each subset. Next, the FD between the three subsets is extracted. We extracted two FD values: between subsets of low and medium quality images (FD1), and between subsets of medium and high quality images (FD2). The quality assessment algorithms are tuned so as to maximize these two FD values.

In Table III it is shown the results of this experimental evaluation procedure. The quality measures are ranked on the basis of the FD1 value. Also, Fig. 5 depicts quality distributions of the three subsets for all the quality algorithms tested. It can be observed from Table III that FD values between subsets of low and medium quality images (FD1) are higher than FD values between subsets of medium and high quality images (FD2) in most cases. This suggests that the tested quality algorithms are specially good at discriminating images of bad quality from the rest of images. However when image quality increases, discrimination capability between quality groups decreases for both sensors. This can also be observed in Fig. 5, where distributions of the medium and high quality subsets are highly overlapped, specially for the optical sensor.

It can also be seen from Table III that all quality algorithms result in higher Fisher distances for the capacitive sensor (i.e., better discrimination between quality groups). There are a number of quality estimation algorithms for the optical sensor that result in high separation between subsets of low and medium quality images (FD1), but most of the algorithms result in low separation between subsets of medium and high quality (FD2). We observe from our experiments that, in general, the discrimination capability is lower for the optical sensor than for the capacitive one.

By looking at Table III, we found interesting relationships between sensor technology and features used for image quality assessment. For instance, quality measures relying on ridge clarity $\left(Q_{L C S}, Q_{A}\right.$ and $\left.Q_{V A R}\right)$ are ranked first for the optical sensor and, on the contrary, they are ranked last for the capacitive one. The opposite happens with measures relying on ridge strength $\left(Q_{O C L}\right.$ and $\left.Q_{G A B O R}\right)$ or ridge continuity $\left(Q_{L O Q}\right.$ and $\left.Q_{C O F}\right)$. Worth noting, quality measures relying on integrity of the ridge-valley structure $\left(Q_{F R E C}\right.$ and $\left.Q_{R V}\right)$ work reasonably well for both sensors. We can also see from these results that quality measures relying on similar features show similar behavior (i.e. they have close positions in the rank). It is worthy to remark that optical sensors are based on light reflection properties [1] which strictly impact on the related grey level values, and that the grey level features-based quality 
measure ranks first for the optical sensor. Therefore, there seems to be a close relationship between the physical properties of the optical sensor and the quality measures that better work with this sensor.

\section{iiiiTable 3 goes here ¿¿¿¿i}

\section{Verification performance improvement}

We now compare the capability of quality measures to improve the verification performance as images scored with bad quality are discarded. In the verification experiments reported in this paper, we use both the publicly available minutiae-based matcher included in the NIST Fingerprint Image Software 2 (NFIS2) [26] and the ridge-based fingerprint matcher developed in the Biometrics Research Lab. at Univ. Autonoma de Madrid, Spain [29]. These two matchers are based on well-known and widely used approaches for fingerprint verification. Minutiae is certainly the most used feature for fingerprint recognition, thanks to its analogy with the way forensic experts compare fingerprints and its acceptance as a proof of identity in the courts of law, and other features have been also proposed in the literature as alternative to minutiae, being the ridge information the most widely studied [1]. It has been found in previous works that the performance of minutiae-based systems depends on the quality of fingerprint images, whereas ridge-based matchers are more robust to image quality degradation $[3,4,29]$, although no studies have taken into account differences between sensors of different technology.

For our evaluation and tests with NFIS2, we have used the following packages: i) MINDTCT for minutiae extraction; and ii) BOZORTH3 for fingerprint matching. MINDTCT takes a fingerprint image and locates all minutiae in the image by means of binarization and thinning, assigning to each minutia point its location, direction, type, and quality. The BOZORTH3 matching algorithm computes a match score between the minutiae from a template and a test fingerprint. A sample fingerprint image, the resulting binary fingerprint and the minutiae pattern superimposed on the thinned image are shown in Fig. 6 . For detailed information of MINDTCT and BOZORTH3, we refer the reader to [26]. The ridge-based matcher uses a set of Gabor filters to capture the ridge strength. The input fingerprint image is convolved with 8 Gabor filters with different directions. The variance of the filter responses across all filtered images is used as feature vector, which is called 
FingerCode because of the similarity to previous research works [30]. The automatic alignment is based on the system described in [31]. No image enhancement is performed since Gabor filters are robust enough to the typical noise present in the fingerprint images. In the matching stage, a similarity score is computed as the Euclidean distance between the two aligned FingerCodes. A sample fingerprint image, the resulting filtered image with a Gabor filter of direction $\theta=0^{\circ}$ and its FingerCode are shown in Fig. 7.

\section{iiiiFigure 6 goes here $i$ $i j i$}

\section{iiiiFigure 7 goes here $¿$ iji}

We consider the 10 impressions from the same finger of the DIEEE data set as enrolment templates. Genuine matchings are obtained comparing the templates to the 10 corresponding impressions from the same finger of the EXTREME data set. Impostor matchings are obtained by comparing one template to the 10 impression of the EXTREME data set from all the other fingers. The total number of genuine and impostor matchings are therefore $42 \times 10 \times 10=4,200$ and $42 \times 41 \times 10=17,220$, respectively, per sensor. We further assign a quality value to each score. The quality of a score is defined as $\sqrt{Q k_{\mathrm{e}} \cdot Q k_{\mathrm{t}}}$, where $Q k_{\mathrm{e}}$ and $Q k_{\mathrm{t}}$ are the image qualities of the enrolled and input fingerprints respectively corresponding to the matching. A quality ranking of the scores is carried out and the EER value is then computed discarding scores with the lowest quality value. Results of this procedure are shown in Figs. 8 and 9. Score quality values have been normalized to lie into the $[0,1]$ range for better comparison between quality measures.

Based on the results of Figs. 8b and 9b, we find close relationship between verification performance improvement and the discrimination capability reported in Sect. III B for the optical sensor. The algorithms with the lowest discrimination capability $\left(Q_{C O F}, Q_{O C L}\right.$, $\left.Q_{L O Q}\right)$ result in the lowest improvement of performance for both matchers. On the other hand, the algorithm ranked first for the optical sensor $\left(Q_{V A R}\right)$ results in an important improvement of performance both for the minutiae- and the ridge-based matcher (a relative improvement of around $60 \%$ and 24\%, respectively, is observed in Figs. 8b and 9b).

For the capacitive sensor, on the contrary, different results are obtained depending on the matcher. It can be seen in Fig. 8a that the verification performance is improved for all the quality measures with the minutiae-based matcher. This is not true with the ridge-based matcher, obtaining a slightly improvement of performance in this case (see Fig. 9a) and even no improvement is observed with the quality measures ranked last in Table III for this 
sensor $\left(Q_{E N E R G Y}, Q_{L C S}, Q_{V A R}, Q_{A}\right)$.

Taking the relative EER improvement into account, we observe from Figs. 8 and 9 that higher improvement is obtained with the optical sensor for both matchers. This can be due to the smaller acquisition surface of the capacitive one and it can also explain the slightly improvement of performance observed with the ridge-based matcher. It is well known that acquisition surface of fingerprint sensors has impact on the performance due to the amount of discriminative information contained in the acquired biometric data [1]. As a result, increasing image quality results in smaller improvement for the capacitive sensor due to this inherent limitation. Or, in other words, degrading image quality has more impact on the performance of the optical sensor, since higher amount of discriminative information is degraded.

\section{iiijFigure 8 goes here $i$ i¿ \\ iiiiFigure 9 goes here ¿i¿i}

\section{CONCLUSIONS}

In this paper, several representative measures for assessing the quality of fingerprint images have been compared, and their differences in behavior when using an optical and a capacitive sensor have been pointed out.

In particular, all quality algorithms have been capable of rejecting images of bad quality for both sensors. However, when image quality is increased, discrimination capability decreases. In general, the discrimination capability is lower for the optical sensor than for the capacitive one. We also pointed out interesting relationships between sensor technology and features used for image quality assessment. The most discriminative measures with one sensor have been the least discriminative ones with the other sensor, and vice-versa. In particular, measures relying on grey level features have been the most discriminative with the optical sensor. We have also compared the performance improvement obtained with each sensor as images with the worst quality are discarded. We have used in our experiments a minutiae- and a ridge-based matcher. For the optical sensor, there is a close relationship between performance improvement and reported discrimination capability for both matchers. On the other hand, no significant performance improvement is obtained for the capacitive sensor when using the ridge-based matcher. 
Future work includes exploiting these differences of behavior in a multi-algorithm or a multi-sensor environment. Having found that quality measures behave differently depending on the sensor, we are also aimed at finding a suitable vector of measures for each sensor that increases the separation between quality groups. Extending this study to a larger set of commercial sensors and also including sensors with other acquisition technology (e.g. thermal ones) is also being considered.

\section{ACKNOWLEDGMENTS}

This work has been supported by Spanish project TEC2006-13141-C03-03, and by European Commission IST-2002-507634 Biosecure NoE. Author F. A.-F. thanks Consejeria de Educacion de la Comunidad de Madrid and Fondo Social Europeo for supporting his PhD studies. Author J. F. is supported by a Marie Curie Fellowship from the European Commission. 
[1] D. Maltoni, D. Maio, A.K. Jain, and S. Prabhakar. Handbook of Fingerprint Recognition. Springer, New York, 2003.

[2] A.K. Jain, A. Ross, and S. Pankanti. Biometrics: A tool for information security. IEEE Trans on Information Forensics and Security, 1:125-143, 2006.

[3] D. Simon-Zorita, J. Ortega-Garcia, J. Fierrez-Aguilar, and J. Gonzalez-Rodriguez. Image quality and position variability assessment in minutiae-based fingerprint verification. IEE Proceedings - Vis. Image Signal Process., 150(6):402-408, December 2003.

[4] J. Fierrez-Aguilar, Y. Chen, J. Ortega-Garcia, and A.K. Jain. Incorporating image quality in multi-algorithm fingerprint verification. Proc. ICB, LNCS-3832:213-220, 2006.

[5] BQW. NIST Biometric Quality Workshop, Gaithersburg, MD, USA, http://www.itl.nist.gov/iad/894.03/quality/workshop07/index.html, 2007.

[6] Raffaele Cappelli, Dario Maio, Davide Maltoni, James L Wayman, and Anil K Jain. Performance evaluation of fingerprint verification systems. IEEE Trans Pattern Anal Mach Intell, 28(1):3-18, Jan 2006.

[7] D. Maio, D. Maltoni, R. Capelli, J.L. Wayman, and A.K. Jain. FVC2002: Second fingerprint verification competition. Proc. ICPR, 3:811-814, 2002.

[8] FVC2006. Fingerprint verification competition - http://bias.csr.unibo.it/fvc2006/default.asp. 2006.

[9] A. Ross and A.K. Jain. Biometric sensor interoperability: A case study in fingerprints. Proc. BioAW, LNCS-3087:134-145, May 2004.

[10] P. Grother and et al. MINEX - Performance and interoperability of the INCITS 378 fingerprint template. NISTIR 7296 - http://fingerprint.nist.gov/minex, 2005.

[11] F. Alonso-Fernandez, R.N.J. Veldhuis, A.M. Bazen, J. Fierrez-Aguilar, and J. Ortega-Garcia. Sensor interoperability and fusion in fingerprint verification: A case study using minutiaeand ridge-based matchers. Proc. ICARCV, Special Session on Biometrics, 2006.

[12] A. Ross and R. Nadgir. A calibration model for fingerprint sensor interoperability. Proc. of SPIE Conference on Biometric Technology for Human Identification III, 2006.

[13] F. Alonso-Fernandez, J. Fierrez-Aguilar, and J. Ortega-Garcia. Sensor interoperability and fusion in signature verification: a case study using tablet pc. Proc. IWBRS, LNCS-3781:180- 
$187,2005$.

[14] C. Vielhauer. Handwriting biometrics: Issues of integration in identification documents and sensor interoperability. SPIE Journal of Electronic Imaging, 15(4), 2006.

[15] F. Alonso-Fernandez, Julian Fierrez, Javier Ortega-Garcia, Joaquin Gonzalez-Rodriguez, H. Fronthaler, K. Kollreider, and J. Bigun. A comparative study of fingerprint image quality estimation methods. IEEE Trans. on Information Forensics and Security (to appear), 2007.

[16] H. Kang, B. Lee, H. Kim, D. Shin, and J. Kim. A study on performance evaluation of fingerprint sensors. Proc. AVBPA, LNCS-2688:574-583, 2003.

[17] N.C. Sickler and S.J. Elliott. An evaluation of fingerprint image quality across an elderly population vis-a-vis an 18-25 year old population. Proc. ICCST, pages 68-73, 2005.

[18] J. Bigun. Vision with Direction. Springer, 2006.

[19] E. Lim, X. Jiang, and W. Yau. Fingerprint quality and validity analysis. Proc. ICIP, pages 469-472, 2002.

[20] Y. Chen, S. Dass, and A.K. Jain. Fingerprint quality indices for predicting authentication performance. Proc. AVBPA, LNCS-3546:160-170, 2005.

[21] L. Shen, A. Kot, and W. Koo. Quality measures of fingerprint images. Proc. AVBPA, LNCS2091:266-271, 2001.

[22] T.P. Chen, X. Jiang, and W.Y. Yau. Fingerprint image quality analysis. Proc. ICIP, pages 1253-1256, 2004.

[23] L. Hong, Y. Wan, and A.K. Jain. Fingerprint imagen enhancement: Algorithm and performance evaluation. IEEE Trans. on PAMI, 20(8):777-789, August 1998.

[24] E. Tabassi, C.L. Wilson, and C.I. Watson. Fingerprint image quality. NIST research report NISTIR7151, August 2004.

[25] E. Tabassi and C.L. Wilson. A novel approach to fingerprint image quality. Proc. ICIP, $2: 37-40,2005$.

[26] C.I. Watson, M.D. Garris, E. Tabassi, C.L. Wilson, R.M. McCabe, and S. Janet. User's Guide to Fingerprint Image Software 2 - NFIS2 (http://fingerprint.nist.gov/NFIS). NIST, 2004.

[27] E. Kukula, S. Elliott, H. Kim, and C. San Martin. The impact of fingerprint force on image quality and the detection of minutiae. Proc. EIT, pages 482-487, 2007.

[28] G.L. Marcialis and F. Roli. Fusion of multiple fingerprint matchers by single-layer perceptron with class-separation loss function. Pattern Recognition Letters, 26:1830-1839, 2005. 
[29] J. Fierrez-Aguilar, L.M. Munoz-Serrano, F. Alonso-Fernandez, and J. Ortega-Garcia. On the effects of image quality degradation on minutiae- and ridge-based automatic fingerprint recognition. Proc. IEEE ICCST, pages 79-82, 2005.

[30] A. Ross, A.K. Jain, and J. Reisman. A hybrid fingerprint matcher. Pattern Recognition, 36(7):1661-1673, July 2003.

[31] A. Ross, K. Reisman, and A.K. Jain. Fingerprint matching using feature space correlation. Proc. BioAW, LNCS-2359:48-57, 2002. 
FIG. 1: Acquisition principles of capacitive and optical sensors.

FIG. 2: Taxonomy of existing fingerprint image quality estimation methods.

FIG. 3: Fingerprint examples from the three subsets of low, medium and high quality. Fingerprint images are plotted at the same scale for: $i$ ) both optical (left subplot) and capacitive sensor; and $i$ ) variable quality (from top to bottom): low, medium, high.

FIG. 4: Optical and capacitive sensor used for the experiments of this paper (Biometrika FX2000 and Precise Biometrics MC100, respectively).

FIG. 5: Quality distributions of the three subsets of images with different quality.

FIG. 6: Processing steps of the minutiae-based matcher. From left to right: original image, resulting binary fingerprint after image enhancement, and minutiae pattern superimposed on the thinned image.

FIG. 7: Processing steps of the ridge-based matcher. From left to right: original image, filtered image with filter orientation $\theta=0$ and FingerCode.

FIG. 8: Minutiae-based matcher.Experiment discarding scores with the lowest quality ( $x$-axis of the different plots). The quality of a score is defined as $\sqrt{Q k_{\mathrm{e}} \cdot Q k_{\mathrm{t}}}$, where $Q k_{\mathrm{e}}$ and $Q k_{\mathrm{t}}$ are the image qualities of the enrolled and input fingerprints respectively corresponding to the matching.

FIG. 9: Ridge-based matcher.Experiment discarding scores with the lowest quality $(x-$ axis of the different plots). The quality of a score is defined as $\sqrt{Q k_{\mathrm{e}} \cdot Q k_{\mathrm{t}}}$, where $Q k_{\mathrm{e}}$ and $Q k_{\mathrm{t}}$ are the image qualities of the enrolled and input fingerprints respectively corresponding to the matching. 
TABLE I: Summary of the quality measures selected for the experiments of this paper.

\begin{tabular}{|c|c|c|}
\hline \hline Quality measure & Property measured & Source \\
\hline \hline$Q_{O C L}$ & Ridge strength & Local angle \\
\hline$Q_{G A B O R}$ & Ridge strength & Gabor filters \\
\hline$Q_{F R E C}$ & Ridge integrity & Local angle \\
\hline$Q_{R V}$ & Ridge integrity & Local angle \\
\hline$Q_{L C S}$ & Ridge clarity & Pixel intensity \\
\hline$Q_{L O Q}$ & Ridge continuity & Local angle \\
\hline$Q_{E N E R G Y}$ & Ridge strength & Power spectrum \\
\hline$Q_{N F I S}$ & Matcher performance & Neural Networks \\
\hline$Q_{A}$ & Ridge clarity & Pixel intensity \\
\hline$Q_{V A R}$ & Ridge clarity & Pixel intensity \\
\hline$Q_{C O F}$ & Ridge continuity & Local angle \\
\hline \hline
\end{tabular}

TABLE II: Conditions of pressure and humidity under which the EXTREME data set was acquired.

\begin{tabular}{|c|c|c||r|r|r|}
\hline \hline Impr. & Press. & Humid. & Impr. & Press. & Humid. \\
\hline \hline 1 & Normal & Normal & 6 & High & Dry \\
\hline 2 & Normal & Normal & 7 & Low & Dry \\
\hline 3 & High & Normal & 8 & Normal & Wet \\
\hline 4 & Low & Normal & 9 & High & Wet \\
\hline 5 & Normal & Dry & 10 & Low & Wet \\
\hline \hline
\end{tabular}


TABLE III: Statistical measures of the three subsets of images with different quality from the EXTREME data set. FD1 (FD2) is the Fisher Distance between subsets of low and medium quality images (medium and high quality images). Quality measures are ranked by FD1

\begin{tabular}{|c|c|c|c|c|c|c|c|}
\hline \multicolumn{4}{|c|}{ CAPACITIVE SENSOR } & \multicolumn{4}{|c|}{ OPTICAL SENSOR } \\
\hline Measure & Property & $F D 1$ & $F D 2$ & Measure & Property & FD1 & $F D 2$ \\
\hline$Q_{G A B O R}$ & Ridge strength & 5,49 & 1,00 & $Q_{V A R}$ & Ridge clarity & 2,64 & 0,41 \\
\hline$Q_{O C L}$ & Ridge strength & 4,44 & 3,21 & $Q_{A}$ & Ridge clarity & 2,53 & 0,45 \\
\hline$Q_{L O Q}$ & Ridge continuity & 4,11 & 0,49 & $Q_{F R E C}$ & Ridge it & 2,16 & 0,16 \\
\hline$Q_{C O F}$ & Ridge continuity & 3,83 & 0,42 & $Q_{R V}$ & Ridge in & 2,14 & 0,27 \\
\hline$Q_{F R E C}$ & Ridge integrity & 3,23 & 0,33 & $Q_{L C S}$ & Ridge clarity & 2,09 & 0,49 \\
\hline$Q_{R V}$ & Ridge integrity & 2,42 & 0,88 & $Q_{G A B O R}$ & Ridge strength & 1,93 & 0,52 \\
\hline$Q_{A}$ & Ridge clarity & 2,35 & 0,55 & $Q_{N F I S}$ & Verification performance & 1,52 & 0,18 \\
\hline$Q_{V A R}$ & Ridge clarity & 2,27 & 0,53 & $Q_{E N E R G Y}$ & Ridge strength & 0,93 & 0,14 \\
\hline$Q_{L C S}$ & Ridge clarity & 2,21 & 0,89 & $Q_{L O Q}$ & Ridge continuity & 0,73 & 0,16 \\
\hline$Q_{N F I S}$ & Verification performance & 2,05 & 0,25 & $Q_{O C L}$ & Ridge strength & 0,73 & 1,68 \\
\hline$Q_{E N E R G Y}$ & Ridge strength & 1,82 & 0,80 & $Q_{C O F}$ & Ridge continuity & 0,60 & 0,22 \\
\hline
\end{tabular}


CAPACITIVE SENSOR

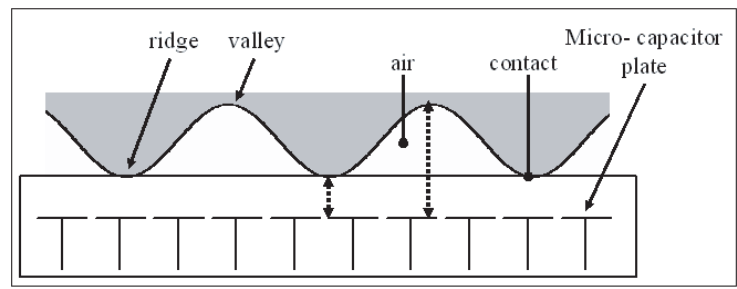

OPTICAL SENSOR

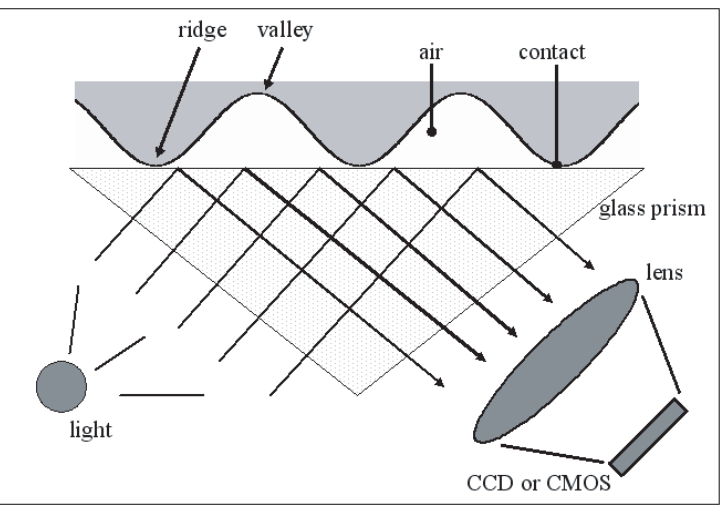

FIG. 1:

Fingerprint Image Quality Estimation Methods
Property
Ridge strength
Ridge continuity
Ridge clarity
Ridge integrity
Source
Orientation field
Gabor filters

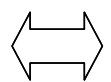
Pixel intensity
Power spectrum
Verification performance
Classifiers

FIG. 2: 


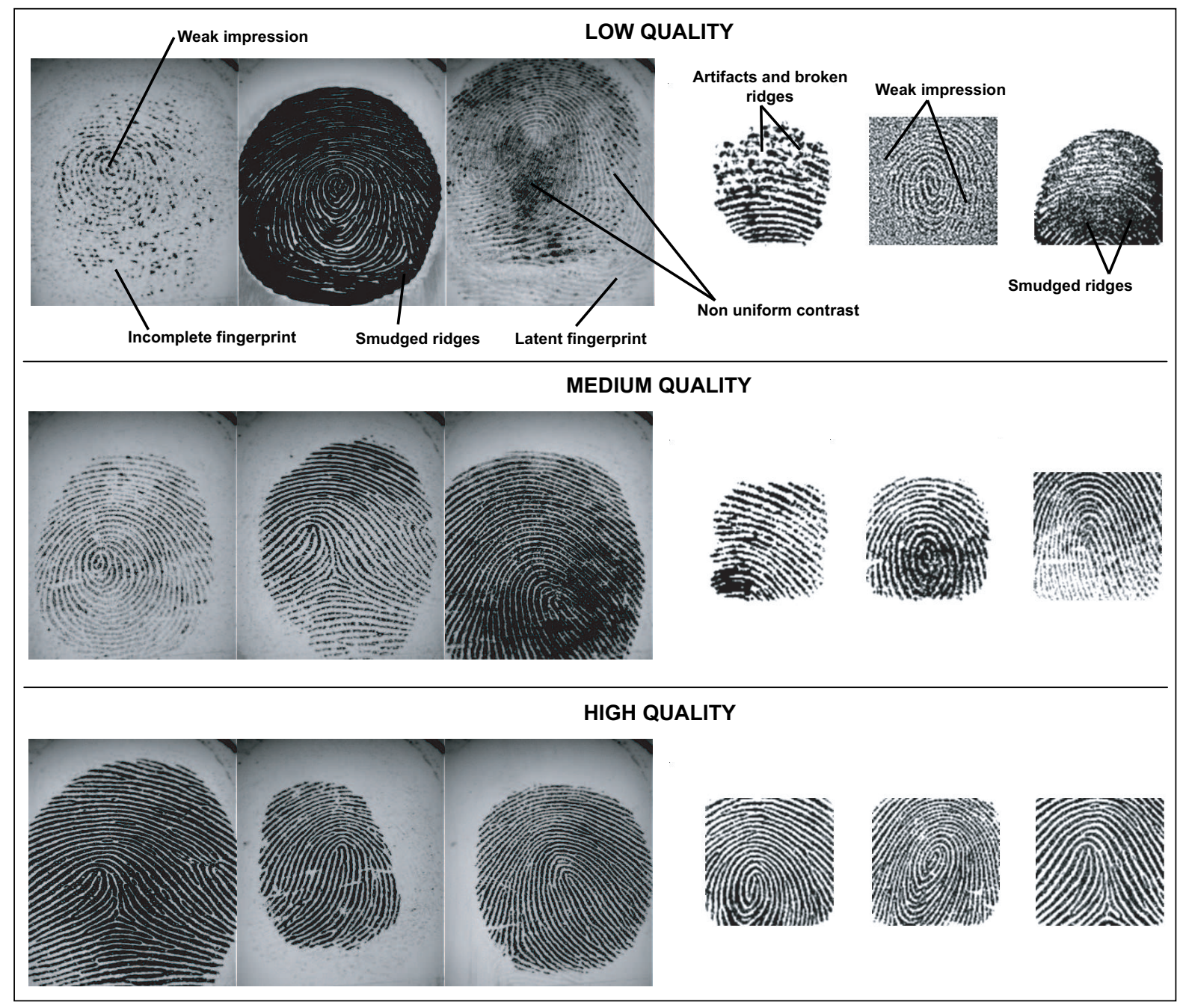

FIG. 3:

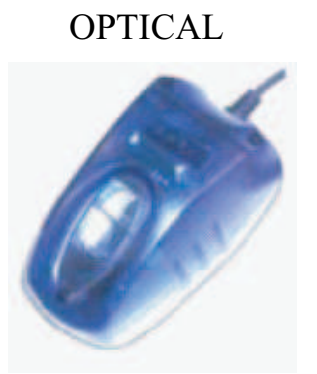

\section{CAPACITIVE}

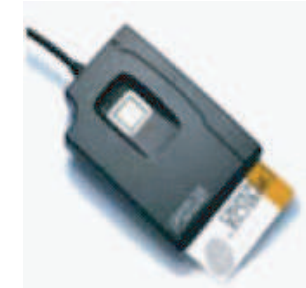

FIG. 4: 

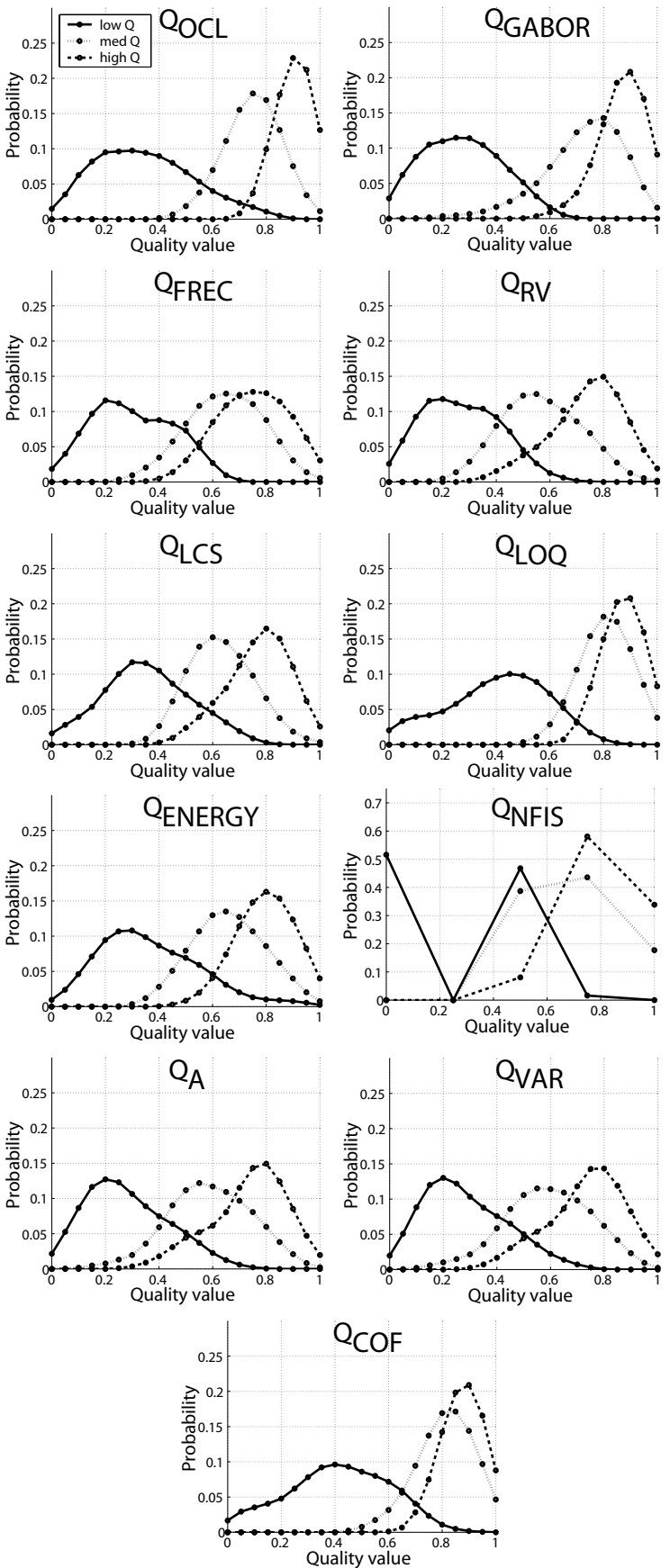

OPTICAL SENSOR
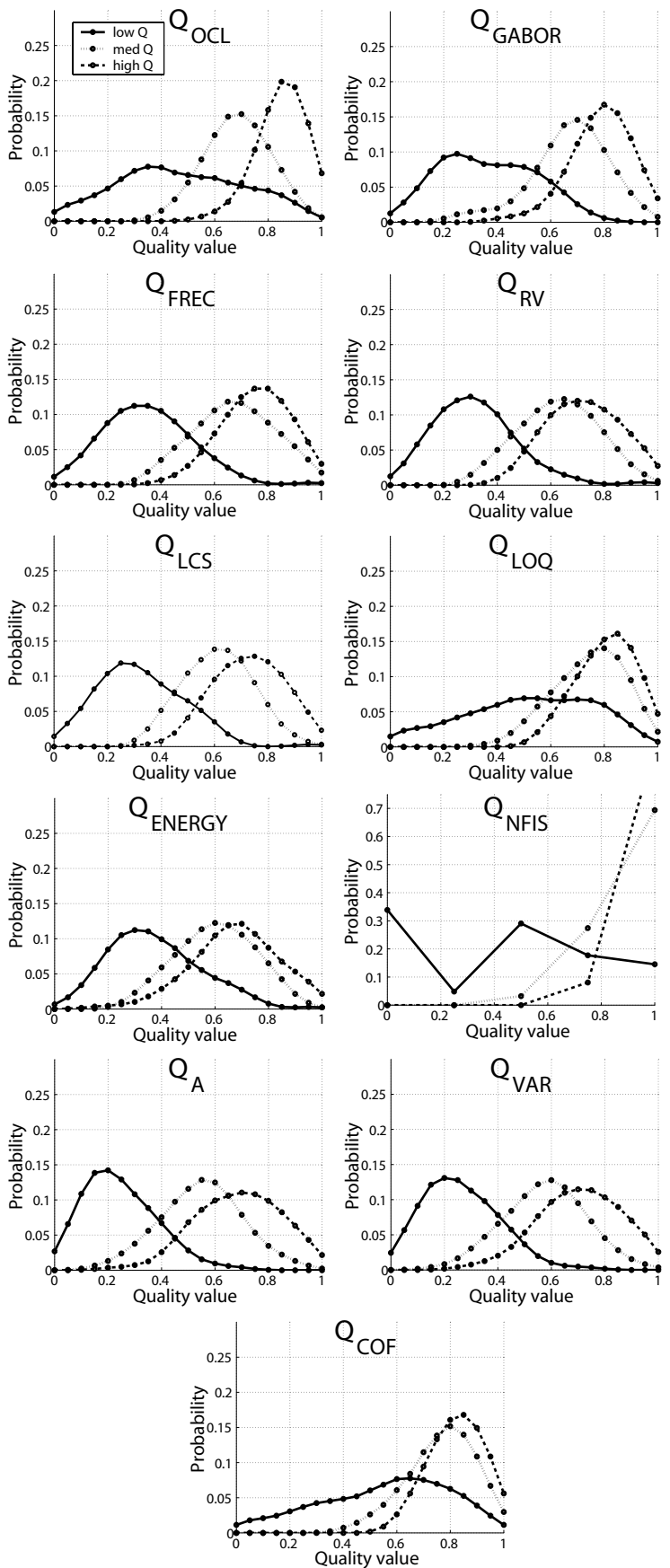

FIG. 5: 


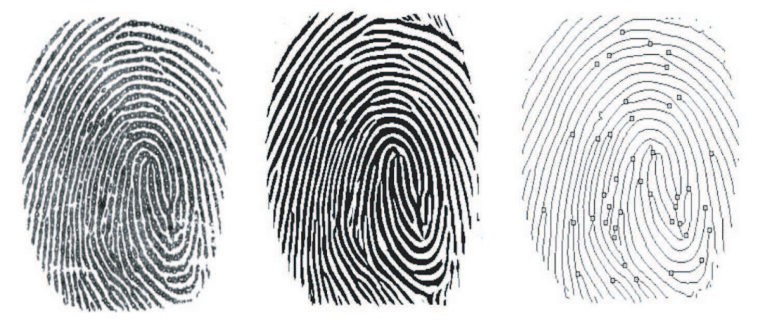

FIG. 6:

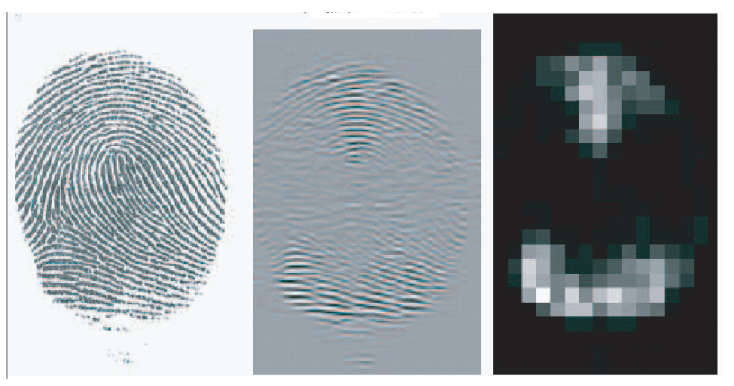

FIG. 7: 

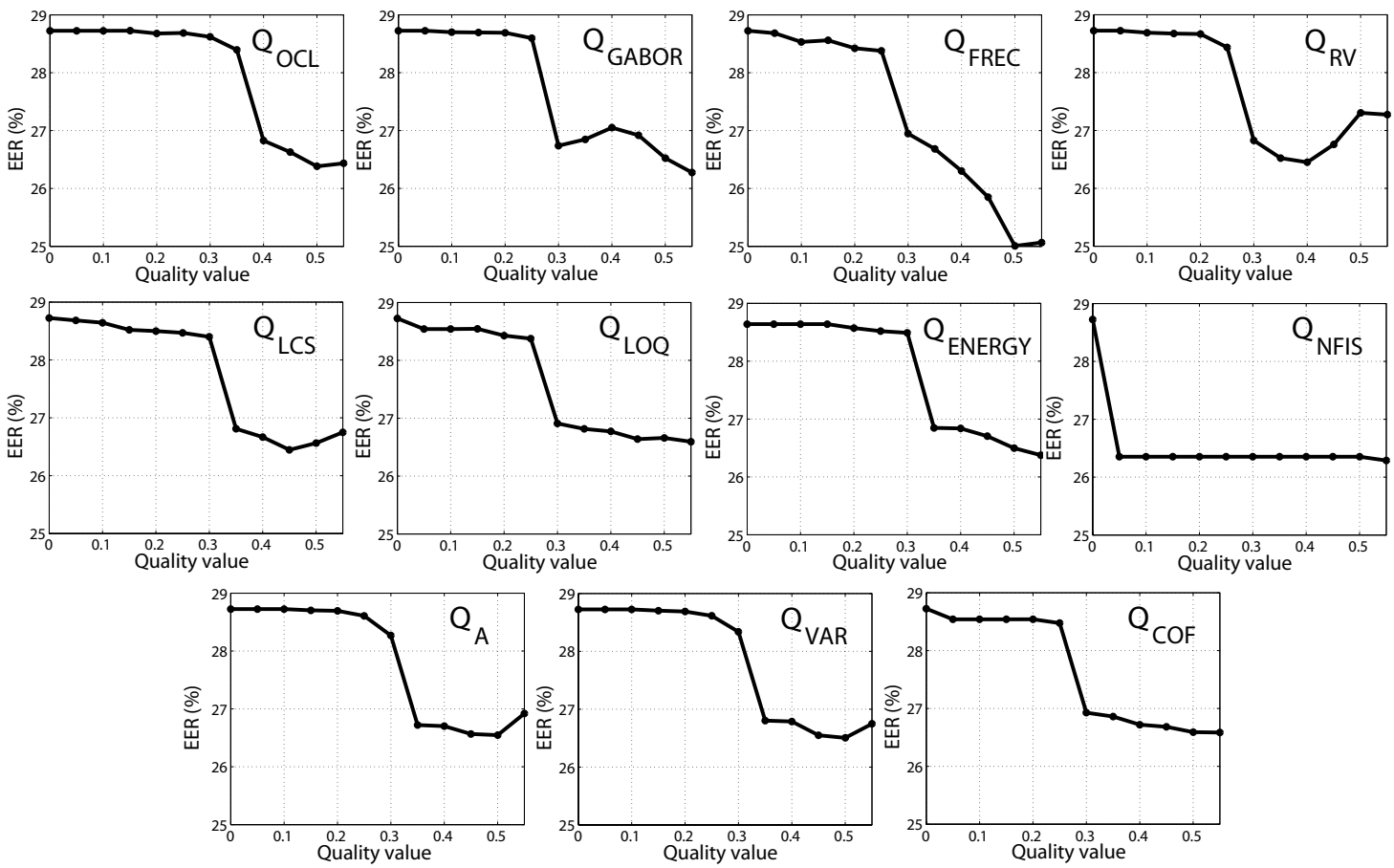

b) OPTICAL SENSOR
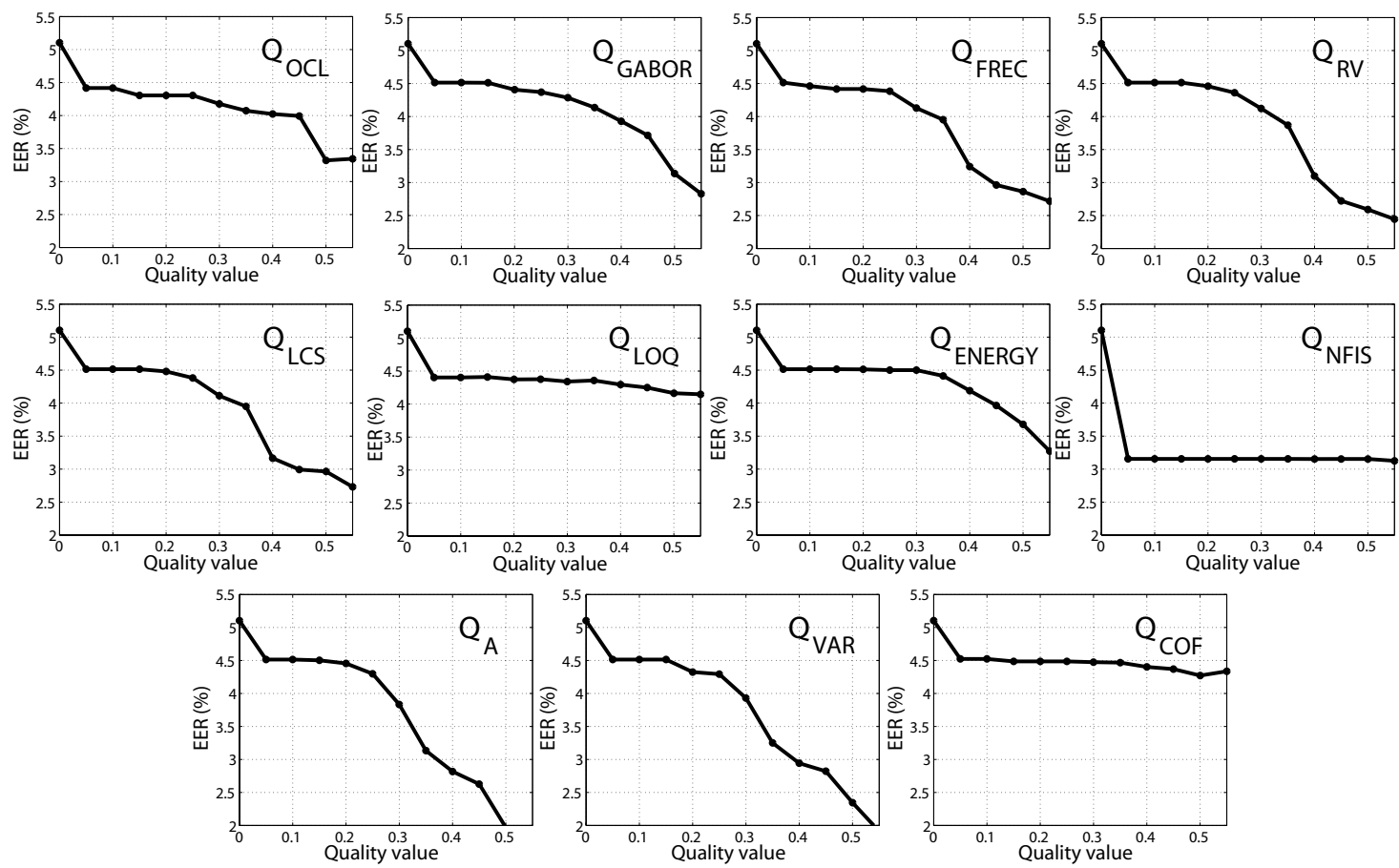

FIG. 8: 
a) CAPACITIVE SENSOR
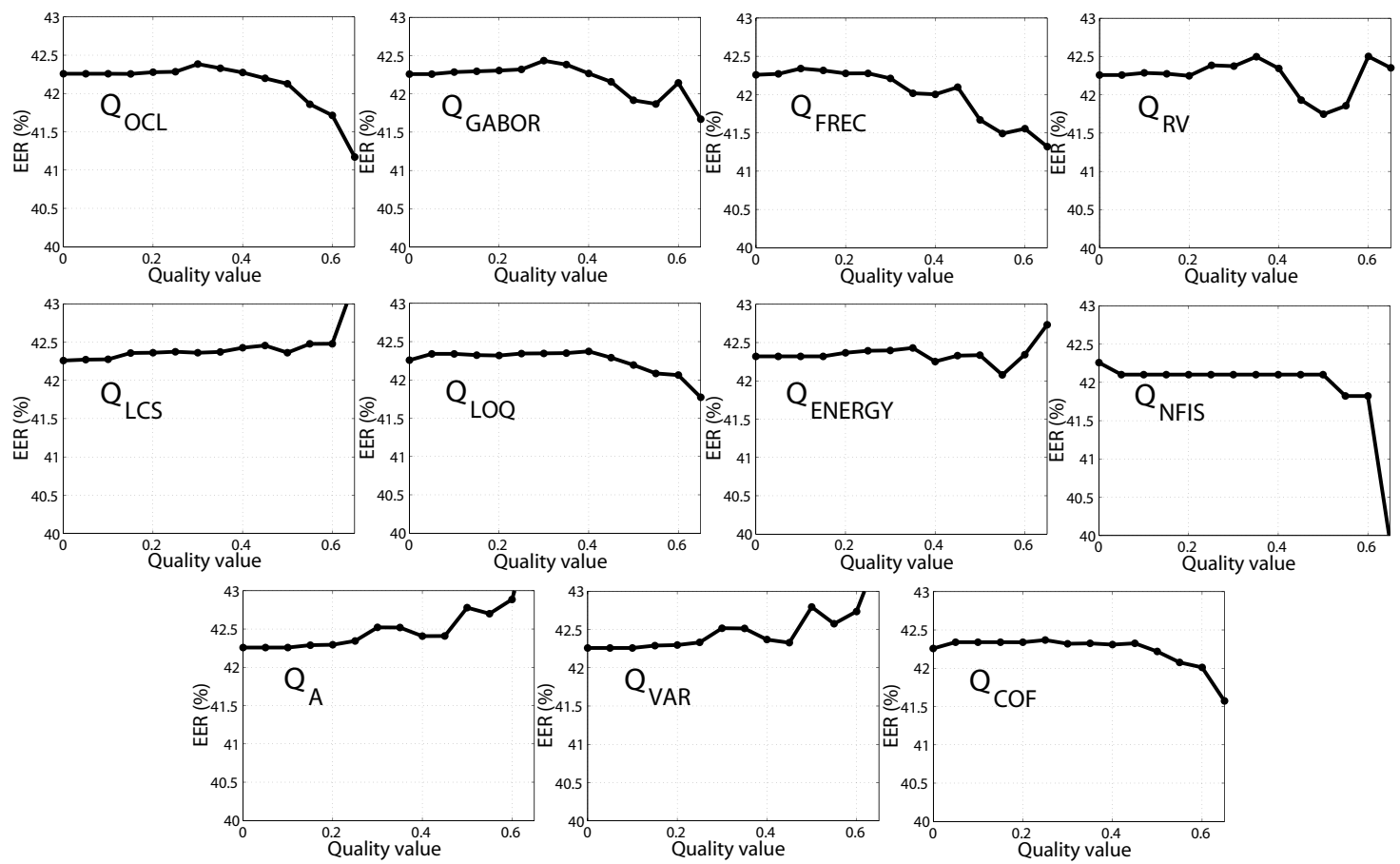

b) OPTICAL SENSOR
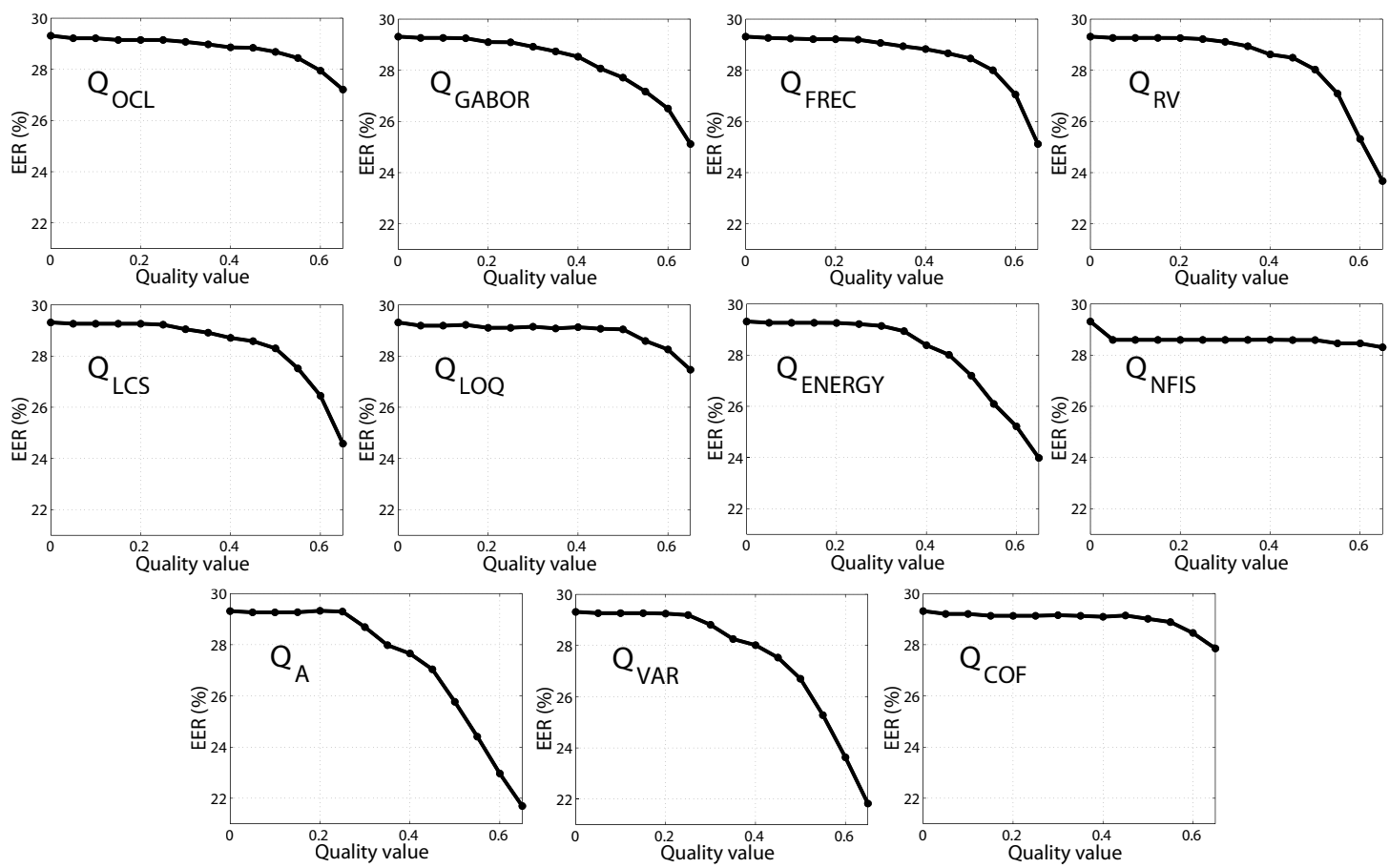

FIG. 9: 